\title{
Sleep disturbance in patients with chronic concussive effects
}

\begin{abstract}
Aim: Sleep disturbance is relatively overlooked in concussion treatment although sleep disorders may prolong or exacerbate symptoms after a concussion. We looked at the incidence of both sleep disturbance and postconcussion symptoms in a sample of recently concussed individuals. Methods \& results: We evaluated scores on the insomnia severity index (ISI) and postconcussion symptom scale (PCSS) in 96 participants with persistent symptoms. Sleep disturbance significantly contributed to the severity of postconcussive symptoms and length of recovery; this effect was less pronounced in athletes. Conclusion: These results suggest a relationship between sleep problems and the time course of recovery from concussive injury. Clinicians who regularly treat concussion would benefit from a more thorough consideration of sleep function in the assessment of postconcussive symptoms.
\end{abstract}

First draft submitted: 5 February 2016; Accepted for publication: 27 April 2016;

Published online: 1 June 2016

Keywords: concussion • PCS • sleep

Postconcussion syndrome (PCS) is a term that describes patients who do not recover within the expected timeframe after concussion [1], which depends upon the patient and the circumstances of injury. PCS is defined by the Diagnostic and Statistical Manual of Mental Disorders, Fourth Edition as cognitive deficits in attention or memory and at least three or more of the following symptoms: fatigue, sleep disturbance, headache, dizziness, irritability, affective disturbance, apathy or personality change that persists for 3 months or longer [2]. Use of a broader definition of PCS (i.e., WHO ICD-10 clinical criteria - persistence of three or more of the following symptoms: headache, dizziness, fatigue, irritability, insomnia, concentration difficulty or memory difficulty) is much more sensitive for identifying PCS patients but does not specify a timeframe [2]. After 3 weeks, patients (especially athletes) begin to worry about when they will recover and by 6 weeks, if symptoms persist, PCS can alter how individuals live their lives [3]. The varying definitions of PCS reflect that PCS is a syndrome characterized by nonspecific cognitive and symptom criteria - it is not a specific diagnosis based upon the evidence of the pathophysiology of concussion [1].

Symptoms associated with disrupted sleep include excessive daytime sleepiness, fatigue, depression, increased pain sensitivity and trouble concentrating [4]. These symptoms clearly overlap with those of PCS. The relationship between concussion and sleep disturbance is not well understood and sleep disturbance is one of the least studied postconcussion symptoms [4]. It has been suggested that many clinicians assume that sleep disturbance holds little consequence for recovery and will decrease as the other symptoms of concussion resolve [4-6]. There is limited literature on the factors that might predispose an individual suffering from a brain injury to sleep disturbance [5]. Recent
Andrea Hinds ${ }^{*, 1}$, Carla R Jungquist ${ }^{2}$, John J Leddy ${ }^{3}$, Fnu Seemant ${ }^{3}$, John G Baker ${ }^{3}$ \& Barry Willer ${ }^{1}$ 'Department of Psychiatry, University at Buffalo, Buffalo, NY, USA ${ }^{2}$ School of Nursing, University at Buffalo, Buffalo, NY, USA

${ }^{3}$ Department of Orthopaedics,

University at Buffalo, Buffalo, NY, USA ${ }^{4}$ School of Social Work,

University at Buffalo, Buffalo, NY, USA

*Author for correspondence:

Tel.: +1 7167963511

alhinds@buffalo.edu 
studies suggest that sleep difficulty is significantly predictive of prolonged recovery from concussion [7]. The lack of normal sleep (total sleep time between 7 and 8 $\mathrm{h}$, with less than $30 \mathrm{~min}$ to get to sleep and less than 30 min awake during the sleep period) [8] could be problematic for recovery and symptom resolution, but little is known about how sleep disturbance could potentially contribute to the exacerbation or persistence of concussion symptoms [9].

There are several types of sleep/wake disorders that may be co-morbid with concussive symptoms, and two main categories of sleep disturbance that may contribute to the persistence of postconcussive effects: sleep disordered breathing such as obstructive sleep apnea (OSA) and insomnia [10]. Although patients with OSA technically fit in the category of insomnia as they meet the diagnostic criteria of difficulty initiating and/or maintaining sleep and suffer from daytime consequences, OSA brings its own set of negative health consequences and is treated differently than insomnia in the absence of OSA [11]. For this reason, screening for sleep disorders usually includes questions about gasping awake during the night, excessive daytime sleepiness and snoring as well as questions more specific to insomnia such as difficulty initiating and maintaining sleep [11].

Other factors that may contribute to the interaction between sleep disturbance and brain injury include age, gender, overall health preinjury and type and severity of trauma. For instance, sleep disturbance is more commonly reported in patients with sportsrelated concussion than in more severe traumatic brain injury [4]. A history of repeated concussive trauma may influence the severity of postconcussive symptoms, including sleep disturbance [4].

As concussive injury and sleep disturbance have potential overlying pathology, it is important to assess their comorbid incidence/prevalence and other factors that may contribute to sleep disturbance after concussion. Establishing co-morbid incidence is the first step in developing evidence on mechanism and treatment. This study was performed to: establish the incidence of sleep disturbance in patients seen at a specialty clinic for concussive injury and, explore the relationship between sleep disturbance and other postconcussive symptoms. We hypothesized that patients with lower sleep quality as measured by the Insomnia Severity Index would exhibit more severe concussion symptoms. We further hypothesized that patients with PCS (as compared with other concussionrelated injuries, such as neck or vestibular injury) would be more likely to report sleep disturbance [12].

\section{Materials \& methods}

The study protocol was approved by the University at Buffalo Institutional Review Board. A retrospec- tive chart review was conducted for 96 patients (both male and female, mean age of 27 years) presenting to the University Sports Medicine Concussion Management center over a 9-month period in 2012. Demographic and diagnostic characteristics of the sample are presented in Table 1.

Patients were selected if they had presented to the clinic for the evaluation of persistent symptoms related to concussive injury. Patients/subjects had been assessed by the attending physician and given a primary diagnosis of postconcussion syndrome, cervicogenic/vestibular/ocular injury, mood or psychiatric symptoms (e.g., anxiety) or postconcussive migraine headache [13] based on symptom presentation, physical examination and exercise intolerance; although each diagnosis shares common symptoms, the primary diagnosis is indicative of the physicianassessed primary cause of symptom persistence (i.e., the primary explanation for a lack of symptom resolution).

Data were extracted from two valid, reliable and commonly used questionnaires - the Insomnia Severity Index (ISI) [5] and the postconcussion symptom scale (PCSS) of the SCAT-2 [14] - completed at the time of evaluation for PCS. No participants reported being diagnosed with, or treated for, sleep dysfunction before their concussive injury; information about pre-existing mood and psychiatric dysfunction was unavailable, but no participants reported receiving treatment for depression or anxiety at the time of injury.

The Insomnia Severity Index (ISI) comprises seven questions that are answered on a $0-4$ scale with higher scores reflecting more severe disturbance. The total score reflects the patient's subjective report of their sleep over the past week. The ISI total score has been shown to consistently correlate with physiological and self-reported measures of sleep quality [5]. Clinically relevant thresholds for insomnia (sleep disturbance) are: none (0-7), mild (8-14), moderate (15-21) and severe (22-28) [5].

The postconcussion symptom scale (PCSS) comprises 22 questions that are answered on a $0-6$ scale with the score reflecting the severity of each symptom. The total score reflects the patient's subjective report of their concussion symptoms at the time the questionnaire is completed. The PCSS has been validated as an assessment tool for the measurement of perceived symptoms associated with concussion. A total score of $\leq 22$ is considered mild, 22-84 moderate and above 84 severe [15].

\section{Analysis}

Descriptive analyses were performed to characterize the sample and compare sleep disturbance severity and sleep 
Table 1. Characteristics of the study sample.

\begin{tabular}{|ll|}
\hline Sample characteristics & Mean (SD), $\mathbf{n}=96$ \\
\hline Age & $27(14)$ \\
\hline Athletic-related concussion & $39.6 \%$ \\
\hline Sex (female) & $43.8 \%$ \\
BMI & $25(4.8)$ \\
\hline Insomnia Severity Index & $10.76(8)$ \\
\hline Postconcussive symptoms & $40.11(28)$ \\
\hline Days since last concussion & $283(537)$ \\
\hline Number of concussions & $1.43(2)$ \\
\hline Primary diagnosis & \\
\hline Postconcussive syndrome & $22.9 \%$ \\
\hline Cervical injury & $21.9 \%$ \\
\hline Both PCS and cervical injury & $35 \%$ \\
\hline Other (psychiatric/headaches) & $19 \%$ \\
\hline PCS: Postconcussion syndrome; SD: Standard deviation. & \\
\hline
\end{tabular}

initiation or maintenance difficulties with concussive diagnostic categories. Regression analysis was conducted to assess associations between sleep disturbance (total ISI score) and concussive symptoms (total PCSS score).

\section{Results}

$57 \%(\mathrm{n}=55)$ of the sample exhibited mild-to-severe sleep disturbance as indicated by a score greater than 7 on the ISI. While there was a slightly higher rate of sleep disturbance in those patients diagnosed with postconcussion syndrome $(64 \%$ of PCS patients reported sleep concerns vs $48 \%$ of those with an alternative diagnosis of cervicogenic or mood dysfunction, or migraine), this difference was not statistically significant. Athletes (as compared with nonathletes) reported significantly lower level of severity of insomnia than nonathletes ( $\mathrm{p}=0.04)$ and athletes were less likely to report mildto-severe insomnia than nonathletes (see Figure 1). Patient sex, age, number of concussions and BMI were not significantly associated with sleep disturbance.

ISI and PCSS were positively correlated $(r=0.65 ; \mathrm{p}$ $<0.001$ ). The regression model to assess predictors for severity of concussive symptoms (age at injury, days since injury, gender and total ISI score) produced a significant finding $\left(\mathrm{R}^{2}=0.430, \mathrm{~F}[4,91]=17.154 ; \mathrm{p}\right.$ $<0.001)$. After controlling for the other variables in the model, the only significant predictor of severity of postconcussive symptoms was severity of sleep-related symptoms $(\beta=0.644 ; \mathrm{p}<0.001)$. Each added point on the ISI increased the PCSS total score by 0.644 . As the PCSS has one question about difficulty sleeping, the model was repeated with the sleep question removed from the total PCSS score. The model results remained significant with strength of sleep relationship only slightly weaker $(\beta=0.616 ; \mathrm{p}<0.001)$.

As a posthoc analysis, we assessed which variables were associated with insomnia severity in this sample, including sex, athletic status, age at injury, days since injury and number of prior concussions. These variables were regressed on ISI total score. The total model was significant $\left(\mathrm{R}^{2}=0.198, \mathrm{~F}[5,90]=4.430\right.$; $\mathrm{p}=0.001)$. Controlling for the other variables in the model, being an athlete $(\beta=-0.244 ; \mathrm{p}=0.042)$ decreased the total ISI score 0.244 . Additionally, every added day since injury increased the total ISI score by $0.264(\beta=0.264 ; \mathrm{p}=0.008)$.

\section{Discussion}

This study showed that chronic sleep disturbance is highly prevalent in patients with postconcussive symptoms and that increased severity of sleep disturbance is associated with severity of postconcussive symptoms. As postconcussive symptoms persist, the likelihood of sleep disturbance increases. Additionally, nonathletes have a higher likelihood of having insomnia after concussion as compared with athletes. The protective effect of athlete status on sleep quality is not clear but may relate to the observation that athletic training is associated with greater parasympathetic activity than in nonathletes $[16,17]$.

These results support the hypothesis that sleep disturbance is a commonly reported occurrence for patients with concussive injury. Sleep problems were reported in more than half of patients suffering persistent concussion-related symptoms and, contrary to our predictions, sleep disturbance was not sig- 


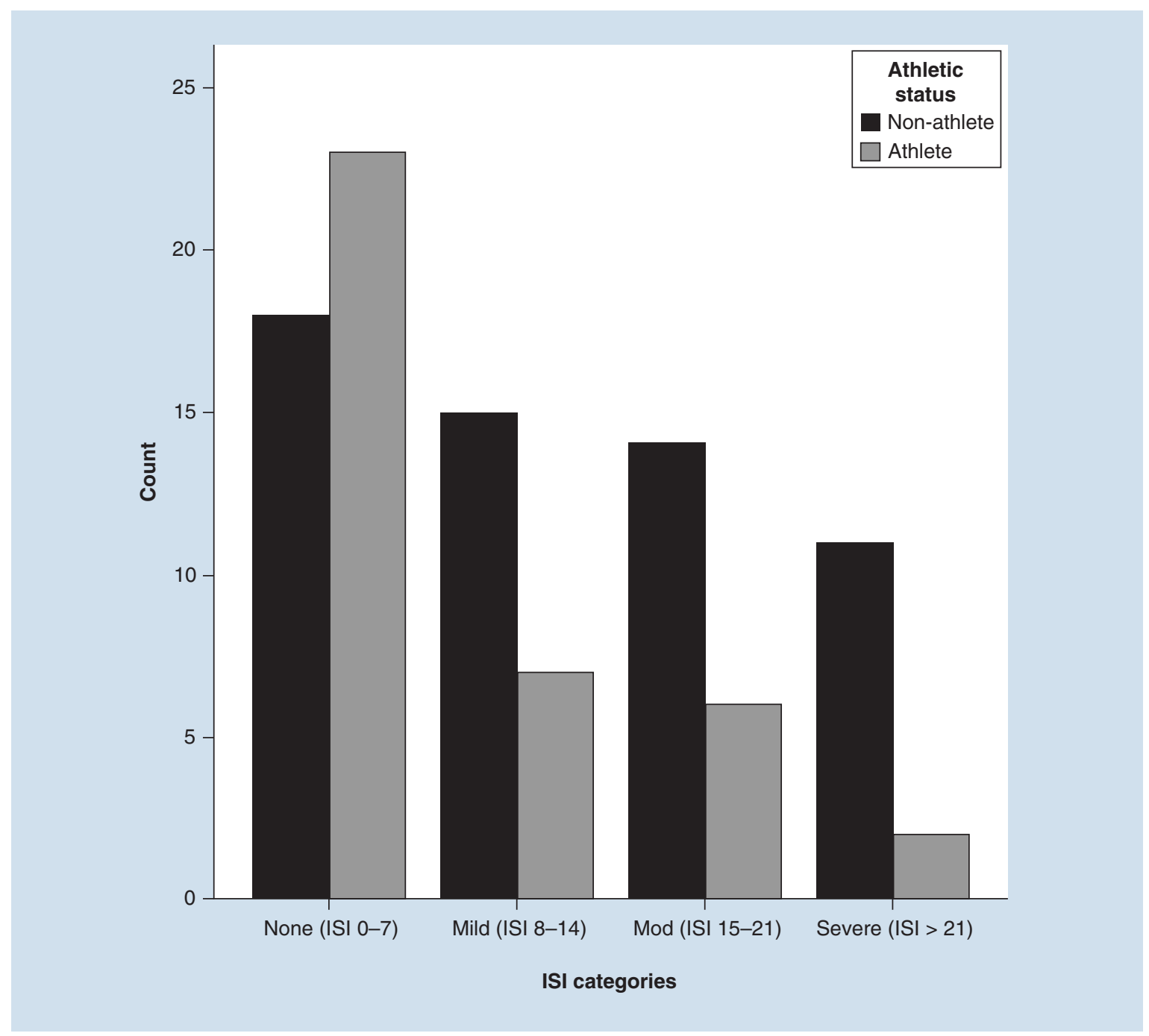

Figure 1. Distribution of Insomnia Severity Index score by severity among athletes $(n=38)$ and nonathletes ( $n=58)$.

ISI: Insomnia Severity Index.

nificantly less prevalent in patients diagnosed with a cervicogenic (neck) injury as opposed to physiologic PCS. While physiologic PCS does appear to elicit or exacerbate sleep-related problems, neck or vestibular symptoms, migraines and mood disturbances appear to be equally associated with poor quality sleep; this may be due to the negative impact of these symptoms (regardless of cause) on quality of life and maintenance of regular routines.

It is also plausible that postconcussion symptoms disrupt normal sleep. A well-known theory of the development of insomnia is that a precipitating factor, such as concussion, occurs and in response to interrupted sleep the patient develops poor sleep habits such as lying in bed awake, reading and watching TV in bed, not keeping a regular bedtime and wake time and/or napping during the day [18]. These poor sleep habits then perpetuate the insomnia [19]. In this study we found that length of time from concussive injury was associated with greater sleep disturbance.

The retrospective design of the study limited the quality and quantity of sleep-related data we were able to gather on each participant; assessment of sleep would have been strengthened with a subjective measure of actual sleep quality, information about the change in sleep quality and quantity over time for each participant, and more comprehensive evaluations of sleep disturbance, including a direct measure of pre-existing sleep dysfunction.

\section{Conclusion}

Our results indicate that athletes and nonathletes report significantly different experiences with sleep disturbance after concussion. Sleep disturbance was also more severe in patients with persistent symptoms, regardless of diagnosis. These results are important for clini- 
cians and patients as severity of sleep disturbance may be associated with delayed recovery. By paying closer attention to sleep-related symptoms, treatment providers may gain a clearer picture of concussion patients' overall health and its impact on prognosis for recovery.

Current treatments for concussion and PCS often do not address sleep concerns and little is known about effective interventions for concussion-related sleep disturbance [20,21]. A comprehensive picture of sleep health may require a more thorough investigation of patient history, taking into account, sleep quality before injury and changes in sleep in relation to the progression of other symptoms over the course of recovery.

The lack of comprehensive research into the relationship between sleep disturbance and concussive injury also indicates a need for more controlled studies to shed light on the many questions that remain. ISI change scores (a comparison of sleep disturbance before and after injury, as well as during treatment) would also provide a more detailed picture of the fluctuation in sleep symptoms in tandem with postconcussion symptoms (such as concentration, fatigue or drowsiness) and quality of life [5]. Self-report and recollection of sleep problems may also be unintentionally inaccurate [22]. Technologies that allow for continuous or regulated sleep reporting at home, or that allow the patient to provide more detailed information about sleep ratings, would reduce bias or recollection difficulties. Examples of such technologies now referred to as devices for ecological momentary assessment are formal actigraphy as well as consumer-friendly sleep tracking devices $[23,24]$.

Knowledge of pre-existing sleep problems before injury would also help to differentiate patients for whom sleep disturbances are brought on by injury from those whose sleep problems are exacerbated. There is the possibility that pre-existing sleep disturbances may themselves have contributed to, or caused, the brain injury [4], and this may have implications for expected recovery. Sleep disturbance in relation to concussion may significantly interfere with recovery and rehabilitation of the patient, and focus on treatments for sleep problems has the potential to significantly impact recovery [20]. It is essential from both a clinical and research perspective to improve our knowledge in this field.

\section{Financial \& competing interests disclosure}

The authors have no relevant affiliations or financial involvement with any organization or entity with a financial interest in or financial conflict with the subject matter or materials discussed in the manuscript. This includes employment, consultancies, honoraria, stock ownership or options, expert testimony, grants or patents received or pending, or royalties.

No writing assistance was utilized in the production of this manuscript.

\section{Ethical conduct of research}

The authors state that they have obtained appropriate institutional review board approval or have followed the principles outlined in the Declaration of Helsinki for all human or animal experimental investigations. In addition, for investigations involving human subjects, informed consent has been obtained from the participants involved.

\section{Open access}

This work is licensed under the Attribution-NonCommercialNoDerivatives 4.0 Unported License. To view a copy of this license, visit http://creativecommons.org/licenses/by-nc-nd/4.0/

\section{Executive summary}

- Patients with concussion often show symptoms of sleep disorders.

- Sleep problems in concussion are not well studied but understanding the relationship between sleep disturbance and concussion has important implications for treatment.

\section{Materials \& methods}

- Chart review was conducted for 96 patients with persistent concussion symptoms.

- Scores for sleep disturbance (Insomnia Severity Index) and concussion symptoms (Postconcussion Symptom Scale) were analyzed.

\section{Results}

- $57 \%$ of patients reported symptoms of sleep disturbance.

- Athletes were significantly less likely to report sleep symptoms.

- Severity of sleep disturbance significantly predicted severity of concussion-related symptoms.

- Days since injury correlated with severity of sleep symptoms were reported.

Discussion \& conclusion

- Sleep disturbance is common in patients with concussion symptoms, regardless of diagnosis.

- There is a need for more comprehensive investigation of the influence of sleep on concussion management, treatment and recovery. 


\section{References}

1 Leddy JJ, Sandhu H, Sodhi V, Baker JG, Willer B. Rehabilitation of concussion and postconcussion syndrome. Sports Health 4(2), 147-154 (2012).

2 Boake C, Mccauley SR, Levin HS et al. Diagnostic criteria for postconcussional syndrome after mild to moderate traumatic brain injury. J. Neuropsychiatry Clin. Neurosci. 17(3), 350-356 (2005).

3 Willer B, Leddy JJ. Management of concussion and postconcussion syndrome. Curr. Treat. Options Neurol. 8(5), 415-426 (2006).

4 Orff HJ, Ayalon L, Drummond SP. Traumatic brain injury and sleep disturbance: a review of current research. J. Head Trauma Rehabil. 24(3), 155-165 (2009).

5 Morin CM, Belleville G, Belanger L, Ivers H. The Insomnia Severity Index: psychometric indicators to detect insomnia cases and evaluate treatment response. Sleep 34(5), 601-608 (2011).

6 Gosselin N, Lassonde M, Petit D et al. Sleep following sportrelated concussions. Sleep Med. 10 (1), 35-46 (2009).

7 Theadom A, Cropley M, Parmar P et al. Sleep difficulties one year following mild traumatic brain injury in a populationbased study. Sleep Med. 16(8), 926-932 (2015).

8 Consensus Conference PWatson NF, Badr MS et al. Joint consensus statement of the American Academy of Sleep Medicine and Sleep Research Society on the recommended amount of sleep for a healthy adult: methodology and discussion. J. Clin. Sleep Med. 11(8), 931-952 (2015).

9 Beaulieu-Bonneau S, Morin CM. Sleepiness and fatigue following traumatic brain injury. Sleep Med. 13(6), 598-605 (2012).

10 Ouellet MC, Beaulieu-Bonneau S, Morin CM. Sleepwake disturbances after traumatic brain injury. Lancet Neurol. 14(7), 746-757 (2015).

11 Qaseem A, Dallas P, Owens DK et al. Diagnosis of obstructive sleep apnea in adults: a clinical practice guideline from the American College of Physicians. Ann. Intern. Med. 161(3), 210-220 (2014).

12 Leddy JJ, Baker JG, Merchant A et al. Brain or strain? Symptoms alone do not distinguish physiologic concussion from cervical/vestibular injury. Clin. J. Sport Med. 25(3), 237-242 (2015).
13 Baker JG, Freitas MS, Leddy JJ, Kozlowski KF, Willer BS. Return to full functioning after graded exercise assessment and progressive exercise treatment of postconcussion syndrome. Rehabil. Res. Pract. 2012, 705309 (2012).

14 Mccrory P, Meeuwisse WH, Aubry M et al. Consensus statement on concussion in sport - the 4th International Conference on Concussion in Sport held in Zurich, November 2012. PMR 5(4), 255-279 (2013).

15 Chen JK, Johnston KM, Collie A, Mccrory P, Ptito A. A validation of the post concussion symptom scale in the assessment of complex concussion using cognitive testing and functional MRI. J. Neurol. Neurosurg. Psychiatry 78(11), 1231-1238 (2007).

16 Carter JB, Banister EW, Blaber AP. Effect of endurance exercise on autonomic control of heart rate. Sports Med. 33(1), 33-46 (2003).

17 Knopfli BH, Bar-Or O. Vagal activity and airway response to ipratropium bromide before and after exercise in ambient and cold conditions in healthy cross-country runners. Clin. J. Sport Med. 9(3), 170-176 (1999).

18 Rao V, Rollings P. Sleep disturbances following traumatic brain injury. Curr. Treat. Options Neurol. 4(1), 77-87 (2002).

19 Spielman AJ, Caruso LS, Glovinsky PB. A behavioral perspective on insomnia treatment. Psychiatr. Clin. North Am. 10(4), 541-553 (1987).

20 Ponsford JL, Ziino C, Parcell DL et al. Fatigue and sleep disturbance following traumatic brain injury-their nature, causes, and potential treatments. J. Head Trauma Rehabil. 27(3), 224-233 (2012).

21 Generali JA, Cada DJ. Modafinil: underarousal, somnolence, and fatigue related to brain injury. Hospital Pharmacy 47(5), 348-350 (2012).

22 Rao V, Spiro J, Vaishnavi $S$ et al. Prevalence and types of sleep disturbances acutely after traumatic brain injury. Brain Inj. 22(5), 381-386 (2008).

23 Evenson KR, Goto MM, Furberg RD. Systematic review of the validity and reliability of consumer-wearable activity trackers. Int. J. Behav. Nutr. Phys. Act. 12(1), 159 (2015).

24 Rosenberger ME, Buman MP, Haskell WL, Mcconnell MV, Carstensen LL. Twenty-four hours of sleep, sedentary behavior, and physical activity with nine wearable devices. Med. Sci. Sports Exerc. 48(3), 457-465 (2016). 